\title{
THE RESISTANCE ON A CIRCULAR CYLINDER IN AN OSCILLATING STREAM*
}

\author{
BY \\ CHANG-YI WANG \\ Massachusetts Institute of Technology
}

\begin{abstract}
The boundary layer equations, formulated in cylindrical polar coordinates, are applied to a circular cylinder in a slightly viscous stream which is oscillating with a high reduced frequency. The resistance is correctly calculated to the second order. The first order part, $45^{\circ}$ out of phase, is due to the interaction of viscosity with acceleration. The second order part, $-90^{\circ}$ out of phase, is due to the interaction of viscosity with curvature. The interaction of viscosity with inertia, which is of second order also, contributes no resistance.
\end{abstract}

1. Introduction. The flow field of a cylinder oscillating perpendicular to the axis in an incompressible fluid which is otherwise at rest is exactly equivalent to the flow field caused by a fixed cylinder in an oscillating stream. Thus the resistance due to viscous shear are the same also. However, the total resistance experienced by the oscillating cylinder is much larger, and is much more difficult to obtain [1]

We will calculate the resistance due to viscous shear, which is also the total force on a fixed cylinder in an oscillating stream. Schlichting [2] was the first to calculate the flow field by applying Blasius' [3] planar boundary layer equations to the oscillating circular cylinder. Through the second order equations Schlichting was able to show the existence of a secondary steady streaming motion which was attributed to the interaction between inertia and viscosity.

Recently the author [4] showed, from a rigorous derivation of the boundary layer equations, that the interaction between viscosity and the curvature of the body cannot be neglected in the higher order equations. Both Blasius and Schlichting, and many other authors who followed their works, may have been incorrect in neglecting these effects of curvature which gives terms of the same order of magnitude as the inertia terms.

2. The boundary layer equations on a circular cylinder. As far as the flow field is concerned, it does not matter whether we fix our coordinates on the cylinder or on the free stream. For simplicity, let us fix the coordinates on the circular cylinder and write the two dimensional Navier-Stokes equations as follows

$$
\begin{aligned}
& \frac{\partial u^{\prime}}{\partial t^{\prime}}+v^{\prime} \frac{\partial u^{\prime}}{\partial r^{\prime}}+\frac{u^{\prime}}{r^{\prime}} \frac{\partial u^{\prime}}{\partial \theta^{\prime}}+\frac{u^{\prime} v^{\prime}}{r^{\prime}} \\
&=\frac{-1}{r^{\prime} \rho^{\prime}} \frac{\partial p^{\prime}}{\partial \theta^{\prime}}+\nu^{\prime}\left[\frac{\partial^{2} u^{\prime}}{\partial r^{\prime 2}}+\frac{1}{r^{\prime}} \frac{\partial u^{\prime}}{\partial r^{\prime}}+\frac{1}{r^{\prime 2}} \frac{\partial^{2} u^{\prime}}{\partial \theta^{\prime 2}}+\frac{2}{r^{\prime 2}} \frac{\partial v^{\prime}}{\partial \theta^{\prime}}-\frac{u^{\prime}}{r^{\prime 2}}\right], \\
& \frac{\partial v^{\prime}}{\partial t^{\prime}}+v^{\prime} \frac{\partial v^{\prime}}{\partial r^{\prime}}+\frac{u^{\prime}}{r^{\prime}} \frac{\partial v^{\prime}}{\partial \theta^{\prime}}-\frac{u^{\prime 2}}{r^{\prime}}=\frac{-1}{\rho^{\prime}} \frac{\partial p^{\prime}}{\partial r^{\prime}}+\nu^{\prime}\left[\frac{\partial^{2} v^{\prime}}{\partial r^{\prime 2}}+\frac{1}{r^{\prime}} \frac{\partial v^{\prime}}{\partial r^{\prime}}+\frac{1}{r^{\prime 2}} \frac{\partial^{2} v^{\prime}}{\partial \theta^{\prime 2}}-\frac{v^{\prime}}{r^{\prime 2}}-\frac{2}{r^{\prime 2}} \frac{\partial u^{\prime}}{\partial \theta^{\prime}}\right],
\end{aligned}
$$

*Received September 22, 1964; revised manuscript received March 16, 1965. This paper was supported by Air Force Grant No. AF-AFOSR-156-64. 


$$
\frac{\partial}{\partial r^{\prime}}\left(r^{\prime} v^{\prime}\right)+\frac{\partial u^{\prime}}{\partial \theta^{\prime}}=0 .
$$

The free stream is assumed to oscillate with a velocity

$$
U^{\prime}=U_{0} \quad \cos \omega t^{\prime}
$$

If we normalize the above equations by the cylinder radius $a$, the frequency of oscillation $\omega$, and the maximum velocity of the free stream $U_{0}$, we find, as in Ref. [1], the flow field is completely characterized by two non-dimensional parameters: a Reynolds number $R$, and a Strouhal number $S$. These are defined as follows:

$$
\begin{aligned}
& R \equiv \frac{U_{0} a}{\nu^{\prime}} \sim \frac{\text { rate of convection by } U_{0} \text { through a distance } a}{\text { rate of diffusion through a distance } a} \\
& S \equiv \frac{a \omega}{U_{0}} \sim \frac{\text { rate of diffusion through the thickness of the boundary layer }}{\text { rate of convection by } U_{0} \text { through a distance } a}
\end{aligned}
$$

$S$ also represents a reduced frequency, and the ratio between the radius of the cylinder to the amplitude of oscillation.

Various simplifications of the Navier-Stokes equations may be obtained by comparing the relative importance of viscous, unsteady, and inertial effects, whose order of magnitudes may be represented respectively by $O(1 / R), O(S)$ and $O(1)$. The most interesting case is when

$$
O(R) \approx O(S)
$$

inside the boundary layer. In this case the mutual balance between viscous and unsteady effects may be more closely examined.

The present problem considers a cylinder in a slightly viscous fluid which is oscillating with a high reduced frequency. The assumptions are

$$
O\left(\frac{1}{R}\right) \approx O\left(\frac{1}{S}\right) \ll 1
$$

The problem may be simplified by expanding the dependent variables in powers of either $1 / S$ or $1 / R$. In order to keep the identity of both, we can develop the following method. Let one of them be equal to a constant multiple of the other, where the multiplication factor is of order unity. Thus we define $\epsilon$ and $\nu$ such that

$$
\frac{1}{\nu R} \equiv \frac{1}{S} \equiv \epsilon \ll 1
$$

where $\epsilon$ is a small number and $\nu$ is a constant of order of one. We can now conveniently expand the dependent variable in terms of $\epsilon$ while regarding $\nu=O(1)$. After the solution is found, we can substitute $\epsilon$ by $1 / S$, and $\nu$ by $S / R$, thus retaining the individuality of both $S$ and $R$.

Keeping this method in mind, we may start afresh to normalize Eqs. (1), (2) and (3). As in Ref. [4], assuming a thin boundary layer, the quantities are normalized by

$$
\begin{aligned}
& t^{\prime}=\frac{t}{\omega}, \quad u^{\prime}=U_{0} u, \quad \nu^{\prime}=\nu_{0} \nu, \\
& \theta^{\prime}=\theta, \quad v^{\prime}=\epsilon U_{0} v, \quad \rho^{\prime}=\rho_{0} \rho, \\
& r^{\prime}=a(1+\epsilon r), \quad p^{\prime}=P_{0} p,
\end{aligned}
$$


where the primed quantities are dimensional, the corresponding unprimed quantities are dimensionless and are of the order of unity, where $P_{0}, \nu_{0}, \rho_{0}$ are defined such that

$$
\begin{aligned}
& \left(\frac{P_{0}}{\rho_{0} U_{0}^{2}}\right) \equiv \frac{1}{\epsilon}, \\
& \left(\frac{U_{0} a}{\nu_{0}}\right) \equiv \frac{1}{\epsilon} \equiv \nu R,
\end{aligned}
$$

and

$$
\frac{1}{\epsilon} \equiv\left(\frac{\alpha \omega}{U_{0}}\right) \equiv S .
$$

Physically, the unsteady forces are balanced by the pressure forces and the viscous forces. They are all of the order of $1 / \epsilon$, as compared with the inertia forces which are of the order of unity.

Then we expand the dependent variables in terms of $\epsilon$

$$
\begin{aligned}
& u=u_{0}+\epsilon u_{1}+0\left(\epsilon^{2}\right), \\
& v=v_{0}+\epsilon v_{1}+0\left(\epsilon^{2}\right), \\
& p=p_{0}+\epsilon p_{1}+0\left(\epsilon^{2}\right) .
\end{aligned}
$$

Substitute into Eqs. (1), (2), (3) and comparing like powers of $\epsilon$, we find the first order equations are not effected by curvature as expected.

$$
\begin{gathered}
\frac{\partial u_{0}}{\partial t}-\nu \frac{\partial^{2} u_{0}}{\partial r^{2}}=-\frac{1}{\rho} \frac{\partial p_{0}}{\partial \theta}, \\
\frac{\partial p_{0}}{\partial r}=0, \\
\frac{\partial v_{0}}{\partial r}+\frac{\partial u_{0}}{\partial \theta}=0 .
\end{gathered}
$$

The second order equations, however, have more terms than the corresponding equations for the planar boundary:

$$
\begin{gathered}
\frac{\partial u_{1}}{\partial t}-\nu \frac{\partial^{2} u_{1}}{\partial r^{2}}=-v_{0} \frac{\partial u_{0}}{\partial r}-u_{0} \frac{\partial u_{0}}{\partial \theta}-\frac{1}{\rho} \frac{\partial p_{1}}{\partial \theta}+\frac{r}{\rho} \frac{\partial p_{0}}{\partial \theta}+\nu \frac{\partial u_{0}}{\partial r}, \\
\frac{\partial p_{1}}{\partial r}=0, \\
\frac{\partial v_{1}}{\partial r}+\frac{\partial u_{1}}{\partial \theta}=-\frac{\partial}{\partial r}\left(r v_{0}\right) .
\end{gathered}
$$

In the third order equations, even the pressure across the boundary layer is not constant any more. It will be futile and meaningless to extend the boundary layer simplification to "third order corrections".

Similarly for the outer potential flow, but without a thin boundary layer assumption, we find,

$$
\frac{\partial U}{\partial t}=-\frac{1}{\rho} \frac{\partial P_{0}}{\partial \theta},
$$




$$
U \frac{\partial U}{\partial \theta}=-\frac{1}{\rho} \frac{\partial P_{1}}{\partial \theta}
$$

on the surface of the cylinder.

The boundary conditions are

$$
\begin{aligned}
& u_{0}=0, \quad u_{1}=0, \\
& v_{0}=0, \quad v_{1}=0, \quad \text { when } r=0 \\
& u_{0}=U . \quad \text { when } r=\infty
\end{aligned}
$$

3. The skin friction. The outer stream, oscillating relative to the cylinder, has a velocity

$$
U^{\prime}\left(\theta, t^{\prime}\right)=2 U_{0} \quad \sin \quad \theta \quad \cos \quad \omega t^{\prime}
$$

on the surface of the cylinder.

Together with Eqs. (4), (5), (6) and (10) the first order solutions are found to be

$$
\begin{aligned}
& u_{0}=\Re\left\{2 \sin \theta e^{i t} \llbracket 1-\exp \left[-(1+i) 2^{-\frac{1}{2}} \eta\right] \rrbracket\right\} \\
& =2 \sin \theta\left[\cos t-\cos \left(t-2^{-\frac{1}{2}} \eta\right) \quad \exp \left(-2^{-\frac{1}{2}} \eta\right)\right], \\
& v_{0}=R\left\{-2 \nu^{\frac{3}{3}} \text { cos } \theta \llbracket\left[\eta+(i-1) 2^{-\frac{1}{2}}\right] e^{i t}+(1-i) 2^{-\frac{1}{2}} \text { exp }\left[-(1+i) 2^{-\frac{1}{2}} \eta+i t\right] \rrbracket\right\} \\
& =-2 \nu^{\frac{3}{4}} \cos \theta\left[\left(\eta-2^{-\frac{1}{2}}\right) \quad \cos t+2^{-\frac{1}{2}} \cos \left(t-2^{-\frac{1}{2}} \eta\right) \quad \exp \left(-2^{-\frac{1}{2}} \eta\right)\right. \\
& \left.+2^{-\frac{1}{2}} \sin \left(t-2^{-\frac{1}{2}} \eta\right) \text { exp }\left(-2^{-\frac{1}{2}} \eta\right)-2^{-\frac{1}{2}} \sin t\right] \text {, }
\end{aligned}
$$

where $\eta=r \nu^{-\frac{1}{2}}$

Notice the solution fails theoretically as $\eta$ goes to infinity because $v_{0}$ approaches infinity also. But good results are expected for the skin friction where $\eta$ approaches zero.

The convective terms on the right side of the second order equations are nonlinear. We must first multiply in the real domain and then transfer to complex variables. Eq. (7) becomes

$$
\begin{aligned}
\frac{\partial u_{1}}{\partial t}-\nu \frac{\partial^{2} u_{1}}{\partial r^{2}}=-2 \sin 2 \theta & \left\{\frac{1}{2} \exp \left(-2^{\frac{1}{2}} \eta\right)-\left[1+i / 2+(1-i) 2^{-\frac{3}{3}} \eta\right] \exp \left[(i-1) 2^{-\frac{1}{2}} \eta\right]\right\} \\
& +2 \sqrt{\nu} \sin \theta\left\{(1+i) 2^{-\frac{1}{2}} \exp \left[-(1+i) 2^{-\frac{1}{2}} \eta\right]-i \eta\right\} e^{i t} \\
& +\sin 2 \theta\left[1+(1+i) 2^{-\frac{1}{3}} \eta\right] \exp \left[-(1+i) 2^{-\frac{1}{2}} \eta\right] e^{2 i t}
\end{aligned}
$$

We assume a solution

$$
u_{1}=2 \sin 2 \theta\left[f(\eta)+g(\eta) e^{2 i t}\right]+2 \nu^{\frac{1}{2}} \sin \theta h(\eta) e^{i t}
$$

Substituting Eq. (16) into Eq. (15) and equating the like harmonics in time, we obtain

$$
\begin{gathered}
\frac{d^{2} f}{d \eta^{2}}=\left[-(1+i / 2)+(i-1) 2^{-\frac{1}{2}} \eta\right] \quad \exp \quad\left[(i-1) 2^{-\frac{1}{2}} \eta\right]+\frac{1}{2} \quad \exp \quad\left(-2^{\frac{1}{2}} \eta\right), \\
\frac{d^{2} g}{d \eta^{2}}-2 i g=\left[-\frac{1}{2}-(1+i) 2^{-\frac{1}{2}} \eta\right] \quad \exp \left[-(1+i) 2^{-\frac{1}{2}} \eta\right], \\
\frac{d^{2} h}{d \eta^{2}}-i h=i \eta-(1+i) 2^{-\frac{1}{3}} \quad \exp \quad\left[-(1+i) 2^{-\frac{1}{2}} \eta\right] .
\end{gathered}
$$


The solution for $u_{1}$ is then

$$
\begin{aligned}
u_{1}=2 & \sin 2 \theta\left\{\frac{1}{4} \exp \left(-2^{\frac{1}{1}} \eta\right)+\left(\frac{1}{2}-2 i\right) \exp \left[(i-1) 2^{-\frac{1}{2}} \eta\right]\right. \\
& \left.-(1+i) 2^{-\frac{3}{3}} \eta \exp \left[(i-1) 2^{-\frac{1}{2}} \eta\right]-\frac{3}{4}\right\}+2 \sin 2 \theta\left\{-\frac{i}{2} \exp [-(1+i) \eta]\right. \\
& \left.+\frac{i}{2} \exp \left[-(1+i) 2^{-\frac{1}{2}} \eta\right]-(i-1) 2^{-\frac{3}{2}} \eta \exp \left[-(1+i) 2^{-\frac{1}{1}} \eta\right]\right\} e^{2 i t} \\
& +2 \nu^{\frac{1}{3}} \sin \theta\left\{-\eta+\frac{\eta}{2} \exp \left[-(1+i) 2^{-\frac{1}{2}} \eta\right]\right\} e^{i t}
\end{aligned}
$$

This solution is identical with those obtained by Schlichting except for the last bracket. The first bracket represents an induced steady secondary motion. The second bracket is an induced subharmonic motion. Both are attributed to the interaction between inertia and viscosity. The third bracket which was neglected by Schlichting, is attributed to the interaction of curvature with viscosity.

The second order continuity equation is also affected by curvature. From Eq. (9), the normal velocity $v_{1}$ is found to be

$$
\begin{aligned}
& v_{1}=-4 v^{\frac{1}{2}} \cos 2 \theta\left\{-2^{-5 / 2} \exp \left(-2^{\frac{1}{3}} \eta\right)+(-3+2 i) 2^{-\frac{1}{2}} \exp \left[(i-1) 2^{-\frac{1}{2}} \eta\right]-\frac{3}{4} \eta\right. \\
& \left.+\frac{i}{2} \eta \exp \left[(i-1) 2^{-\frac{1}{2}} \eta\right]+13 \cdot\left(2^{-5 / 2}\right)\right\}-4 \nu^{\frac{1}{2}} \cos 2 \theta e^{2 i t}\left\{\frac{1}{4}(1+i) \exp [-(1+i) \eta]\right. \\
& \left.+\frac{i}{2} \eta \exp \left[-(1+i) 2^{-\frac{1}{2}} \eta\right]-\frac{1}{4}(1+i)\right\}+2 \nu \cos \theta e^{i \iota}\left\{\frac{3}{2} \eta^{2}+(i-1) 2^{-\frac{1}{2}} \eta\right. \\
& \left.-\frac{i}{2} \exp \left[-(1+i) 2^{-\frac{1}{3}} \eta\right]+3(1-i) 2^{-\frac{1}{2}} \eta \exp \left[-(1+i) 2^{-\frac{1}{2}} \eta\right]+\frac{i}{2}\right\}
\end{aligned}
$$

The surface stress, in cylindrical polar coordinates, is

$$
\tau_{s}=\mu\left[r^{\prime} \frac{\partial}{\partial r^{\prime}}\left(\frac{u^{\prime}}{r^{\prime}}\right)+\frac{1}{r^{\prime}} \frac{\partial v^{\prime}}{\partial \theta^{\prime}}\right]_{r^{\prime}=a}=\left(\rho^{\prime} U_{0}^{2}\right) \nu\left[\frac{\partial u_{0}}{\partial r}+\epsilon \frac{\partial}{\partial r}\left(u_{1}-r u_{0}\right)+0\left(\epsilon^{2}\right)\right]_{r=0}
$$

Substitute the values of $u_{0}$ and $u_{1}$ into Eq. (19) $\tau_{\text {s }}=\left(\rho^{\prime} U_{0}^{2}\right)\left[\nu^{\frac{3}{2}} \sin \theta(\cos t-\sin t)+\epsilon \nu^{\frac{1}{2}} 2^{-\frac{1}{2}} \sin 2 \theta\right.$

$$
\left.+\epsilon \nu^{\frac{1}{2}}\left(2^{\frac{1}{3}}-1\right) \sin \theta(\cos 2 t+\sin 2 t)-\epsilon \nu \sin \quad \theta \quad \cos t+0\left(\epsilon^{2}\right)\right]
$$

Transforming back into dimensional quantities, we find

$$
\begin{aligned}
\tau_{\text {. }}=\left(\rho^{\prime} U_{0}^{2}\right)\left[( \frac { S } { R } ) ^ { \frac { 1 } { 2 } } \operatorname { s i n } \theta \left(\cos \omega t^{\prime}\right.\right. & \left.-\sin \omega t^{\prime}\right)+(R S)^{-\frac{1}{2}}\left(2^{\frac{1}{2}}-1\right) \sin 2 \theta\left(\sin 2 \omega t^{\prime}+\cos 2 \omega t^{\prime}\right) \\
& \left.+(R S)^{-\frac{1}{3}} 2^{-\frac{1}{2}} \sin 2 \theta-\frac{1}{R} \sin \theta \cos \omega t^{\prime}+0\left(\epsilon^{2}\right)\right]
\end{aligned}
$$

We see that the local shear on the surface of the cylinder has a steady part in addition to harmonic and subharmonic parts.

The velocity profile of $u$ at $\theta=90^{\circ}$ for two values of $(R S)^{-\frac{1}{1}}$ is shown in Figs. 2 and 3. Notice how our second order solution fits into the potential profile. 


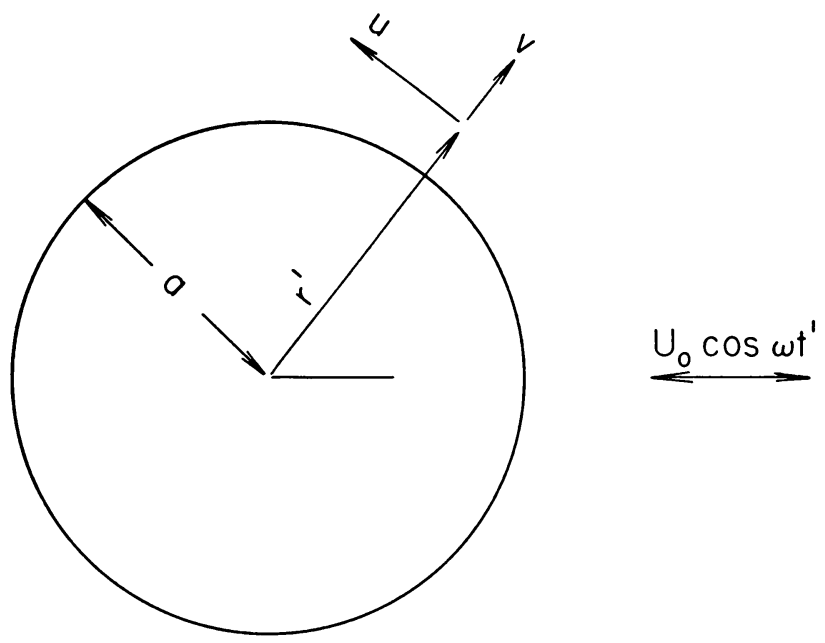

Fig 1. The Coordinate System

4. The resistance on a circular cylinder in an oscillating stream. Since the boundary layer is assumed to be very thin, the total pressure forces will sum up to be zero by potential theory. Denoting the total resistance by $F^{\prime}$ and normalizing we obtain $\frac{F^{\prime}}{\pi\left(\rho^{\prime} U_{0}^{2}\right) a}=F=\frac{2 \int_{0}^{n} \tau_{s} a \sin \theta d \theta}{\pi\left(\rho^{\prime} \nu_{0}^{2}\right) a}=\sqrt{\frac{S}{R}}\left(\cos \omega t^{\prime}-\sin \omega t^{\prime}\right)-\frac{1}{R} \cos \omega t^{\prime}+0\left(\epsilon^{2}\right)$

The first term on the right hand side is of the order of unity. It represents the re-

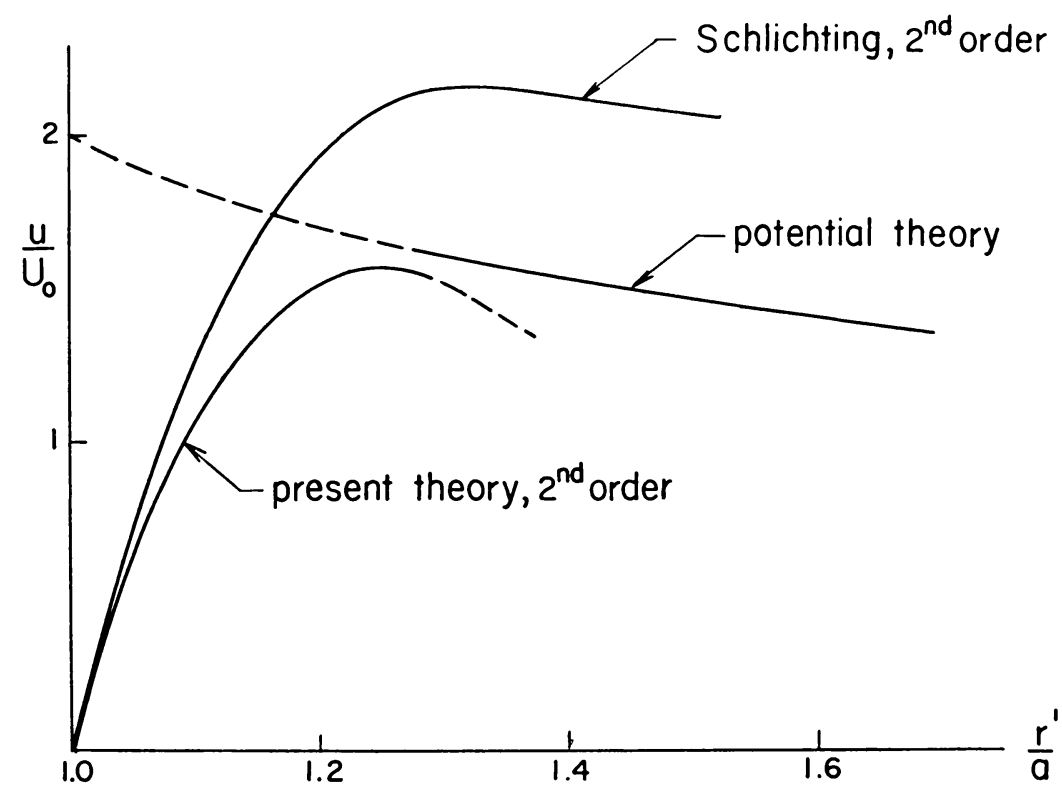

Fig. 2. The Tangential Velocity Profile at $\theta=90^{\circ}$ and $t=0$ with $1 / \sqrt{R S}=0.1=\sqrt{\nu \epsilon}$ 


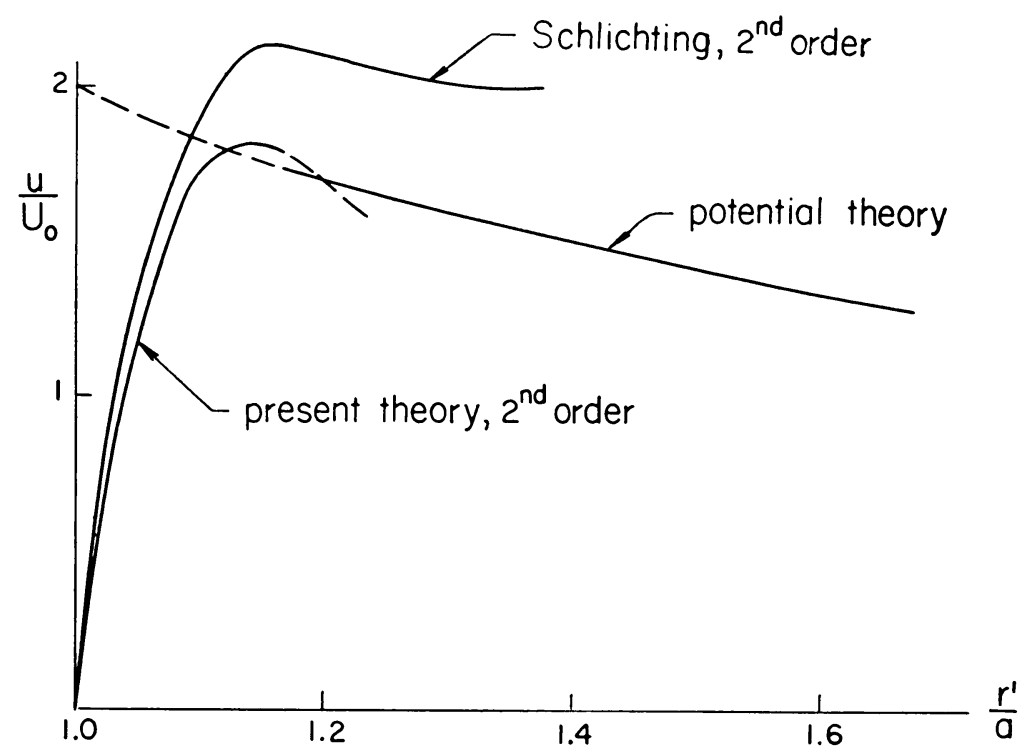

Frg. 3. The Tangential Velocity Profile at $\theta=90^{\circ}$ and $t=0$ with $1 / \sqrt{R S}=0.05$

sistance from the interaction of acceleration with viscosity and leads $\pi / 4$ in phase. The second term is of the order of $\epsilon$. It represents the resistance due to the interaction of curvature with viscosity and lags $\pi / 2$ in phase. Notice the interaction between inertia with viscosity does not contribute to resistance. Other terms are of the order of $\epsilon^{2}$.

Equation (21) can also be written in the form

$$
F=-\sqrt{\frac{S}{R}} \sin \omega t^{\prime}-\left(\frac{1}{R}-\sqrt{\frac{S}{R}}\right) \quad \cos \omega t^{\prime}+0\left(\epsilon^{2}\right)
$$

where the second term, which always opposes the motion of the stream, represents damping.

5. Discussion. Segel [5] also suggested an optimum coordinate system for the asymptotic expansion to get the governing equations. But his method is based on a Stokes approximation, which is linear. One should be careful in obtaining higher order solutions from Segel's method. The assumptions are

$$
\frac{1}{S} \ll \frac{1}{R S} \ll 1
$$

which implies very very small Reynolds number. However, the effects of curvature, though not explicitly mentioned, are also shown in his efforts to obtain a uniformly valid solution.

From an unpublished work of the author the same results for skin friction, Eq. (20), were obtained by a more tedious method of inner and outer expansions. This method is very powerful in solving the total velocity field, for example the acoustic streaming induced by the oscillation. In such cases the boundary layer theory outlined in this paper is inferior in comparison. The inner and outer expansions method applied to the case of an oscillating sphere can be found in Ref. [6]. 


\section{REFERENCES}

1. L. Rosenhead, ed., Laminar boundary layers, Oxford University Press, Great Britain, 1963

2. H. Schlichting, Berechnung ebener periodischer Grenzschichtströmungen Physikalische Z. 33, (1932) 97.

3. H. Blasius, Grenzschichten in Flüssigkeiten mit kleiner Reibung, Zeit. Math. u. Phys. 56 (1908) 1, NCA TM No. 1256

4. C. Y. Wang, On the separation time of the flow past a circular cylinder which is started from rest, to be published in J. Math. and Phys. (1965)

5. L. A. Segel, $A$ uniformly valid asymptotic expansion of the solution to an unsteady boundary layer problem, J. Math. and Phys. 39 (1960) 189

6. C. Y. Wang, The flow induced by an oscillating sphere, J. of Sound and Vibration, 2 (3), 257 (1965) 